\title{
Real-Time Surgical Simulation with Haptic Sensation as Collaborated Works between Japan and Germany
}

\author{
Naoki Suzuki ${ }^{1}$, Asaki Hattori ${ }^{1}$, Shigeyuki Suzuki ${ }^{2}$, Max P. Baur ${ }^{3}$, \\ Andreas Hirner ${ }^{3}$, Susumu Kobayashi ${ }^{4}$, Yoji Yamazaki ${ }^{4}$, Yoshitaka Adachi ${ }^{5}$ \\ 1 Institute for High Dimensional Medical Imaging, Jikei Univ. School of Med. \\ 4-11-1 Izumi-honcho, Komae, Tokyo 201-8601 JAPAN \\ nsuzuki@jikei.ac.jp \\ 2 School of Science and Engineering, Waseda Univ., 3 Bonn Univ., \\ 4 Dept. of Surgery, Jikei Univ. School of Med., 5 Suzuki Motor Corp, R\&D Center
}

\begin{abstract}
As part of an application of tele-virtual surgery and a force feedback device, surgeons in Japan and Germany examined a hepatectomy simulation system. Surgeons in each country could be performed various surgical maneuvers upon the same patient by using our system. Surgeons palpated abdominal skin, made electrical scalpel incisions and widened the incision line by using surgical tools in virtual space. The user can convey tactile sensations by the force feedback device while surgeons performed a virtual operation. Two graphic workstations of equal capability and force feedback devices were engaged in each location. As each workstation communicated only event signals through an ISDN $(64 \mathrm{~Kb})$ line, it made possible to obtain real time televirtual surgery without a large capacity communication infrastructure.
\end{abstract}

\section{Preface}

Up to the present, three dimensional images reconstructed from MRI or CT images used in various applications. In our future medical treatments, the application of virtual reality (VR) techniques to $3 \mathrm{D}$ images has a large potential through telediagnosis and tele-medicine.

We report the results of a tele-virtual surgery experiment between Japan and Germany in this paper. Apparatus at both sites were connected by an ISDN line and equipped with a VR surgery system with a force feedback function. Having this system, surgeons in each plane shared identical tactile sensations.

\section{Surgical Simulation System}

A surgical simulation system has the useful application in the medical field. The system allows a user to repeatedly perform virtual surgery until a suitable procedure is established. This is especially useful for educational medical training.

We have been developing a virtual surgical simulation system upon the following requirements:

W. Niessen and M. Viergever (Eds.): MICCAI 2001, LNCS 2208, pp. 1015-1021, 2001.

(C) Springer-Verlag Berlin Heidelberg 2001 
1). The system should provide a design for the user and determine surgical procedures based on 3D model reconstructed from the patient's data.

2). The system must transmit authentic tactile sensations to the user during organ manipulations by using force feedback device.

In our system, the surgeon (user) is able to perform various surgical maneuvers with

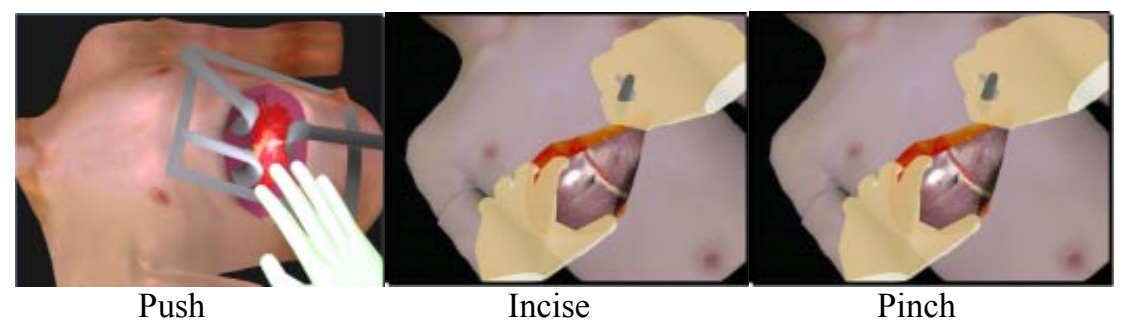

Fig. 1. Application of surgical simulation used in this experiment

suitable surgical tools as interactive actions in a virtual space. This system allows the surgeon to make a scalpel incision, widen the incision line and secure it with forceps (Fig.1). All surgical procedures on a 3D object in a virtual space are proceeded in real-time. Also 3D human structures are reconstructed from 3D patient data that gives such anatomical characteristics as vascularity. Numerical parameters such as location, depth, direction to the targeted organ and excised tissue volumes are measurable with quantitative accuracy.

Various viewpoints such as scale and angles can be displayed the basic system function. It can also alter the transparency of an organ's multiple layers. Rendering by wire frames is also possible. In addition, each organ model is shaded by light sources set in the system's space and separated by easily distinguishable colors. In order to determine possible incision points, these models are texture mapped by using images of the patient's skin and extracted organ texture.

\section{Force Feedback Device}

A haptic device, which giving authentic tactile sensations for the operator, was confirmed very recently. We also have been developing a force feedback device which possesses 16 degrees-of-freedom (DOF) for manual interactions with virtual environments. Followings are summarization of the features of the device manufactured for our virtual surgery system.

1) The force feedback system is composed of two types of manipulators: a force control manipulator and a motion control manipulator.

2) Three force control manipulators are attached to the end of the motion control manipulator.

3) Both ends of each force control manipulator are attached to the thumb, forefinger, and middle finger of the operator.

4) The force control manipulator has a joint structure with minimal inertia and less friction.

5) The motion control manipulator has mechanical stiffness. 
Fig.2 shows the device for the right hand. The three force control manipulators are mounted at the pointed end of this device. These manipulators are attached to user's thumb, forefinger, and middle finger respectively. Fig.3 illustrates the user's fingers are attached to the manipulators. A user and the device attached to both hands were shown in Fig.4. These left and right force feedback devices have the same internal structure. The force feedback device for the right hand is a mirror image of the left one. These devices communicate data (finger location etc.) with the surgical simulation system through a LAN. As soon as an interaction occurs between the user's fingers and a 3D object in a virtual space, a force parameter of tactile sensations calculated by the surgical simulation system, is transferred to these devices. This allows the user to experience tactile sensations in each finger.

\section{Tele-Surgical Simulation System}

Since we intended to examine televirtual surgery without a large capacity communication infrastructure, we used a $1 \mathrm{ch}$ ISDN line $(64 \mathrm{~Kb} / \mathrm{s})$, in this experiment. However, it was difficult to transfer images of simulation results to each location in real-time. Therefore, we installed a simulation program and the patient's 3D modeling into each system. The MRI images produced 3D data of the skin surface, liver, liver vessels, liver tumor and colon. The system transmitted and received only event signals related to the simulation.

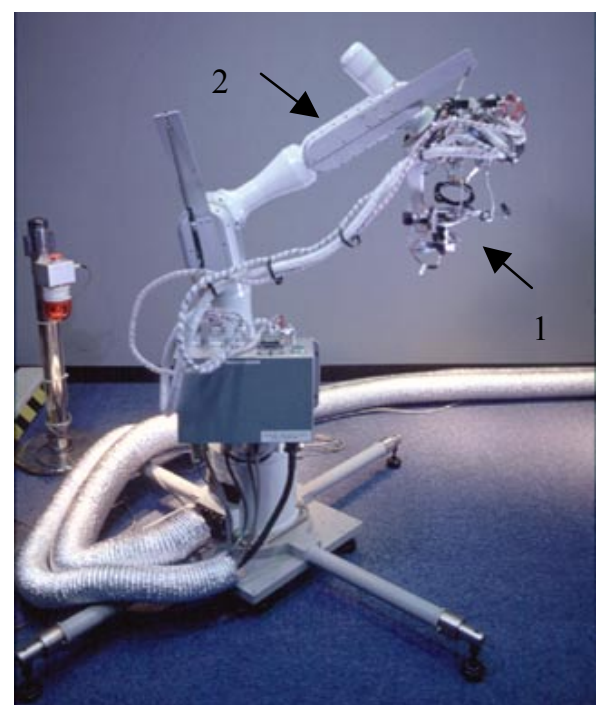

$\mathrm{a}$

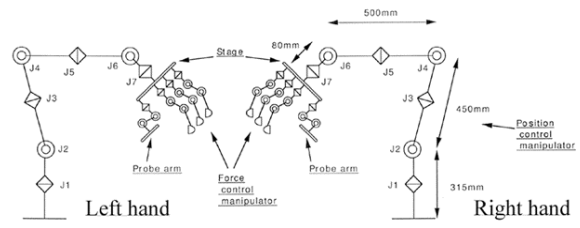

b

Fig. 2. A view of the force feedback device and the block diagram

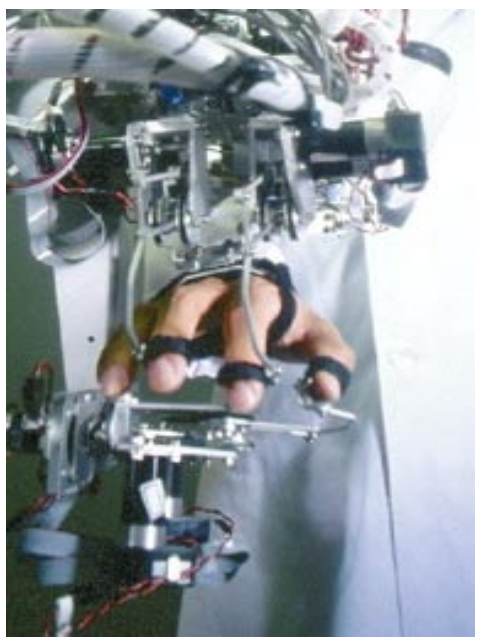

Fig. 4. Force feedback device attached to fingers of the right hand 
The event signal included force feedback device location data, the application's GUI event (buttons, sliders etc.) and calculated force of the force feedback device. The size of data per event is about 200 bytes. In this way, both sites were able to observe an identical simulation result in real-time.

Fig.5 shows the system's outline. For the surgical simulation and teleconference, participants at each location employed two graphic workstations (Japan site: Octane, Indy, German site: Octane, O2. All workstations are SGI inc. products). These workstations were connected with an ISDN line via an ISDN

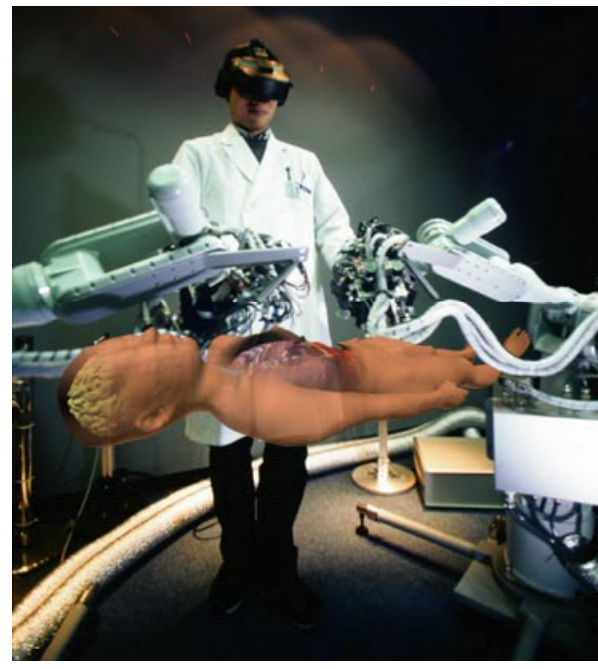

Fig. 6. User with force feedback device attached to both hands

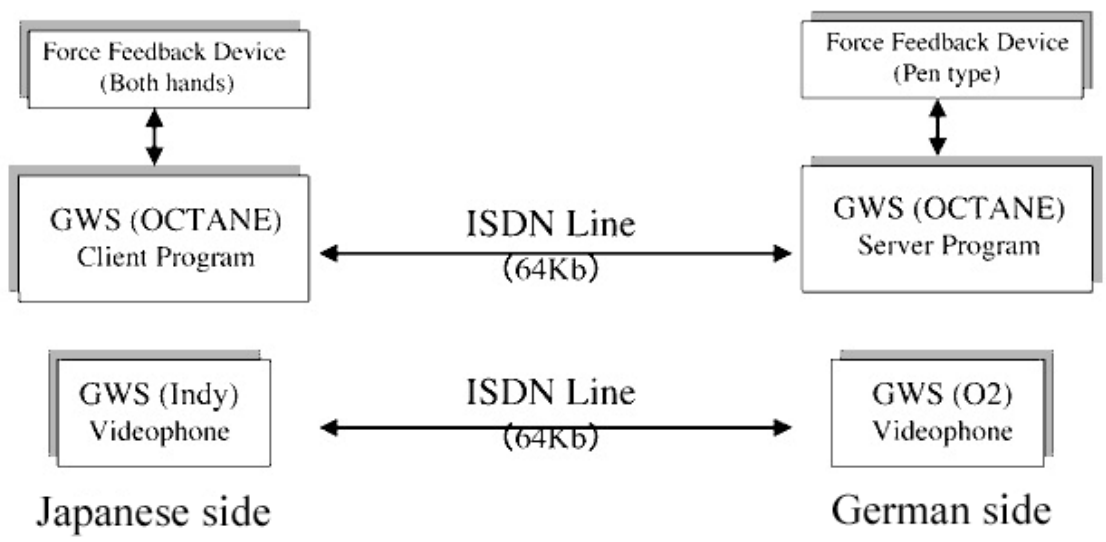

Fig. 5. System outline of tele-virtual surgery

router. A force feedback device was prepared at each workstation. The force feedback was a glove type device attached to both hands in Japan. On the other hand, a pen type device was used in Germany. During the virtual surgical operation, these devices conveyed tactile sensations to the surgeons.

For communicating between each site, we used a teleconference application InPerson (SGI inc.) and video image and audio functioning at 300x200 pixels. This application's video frame was about 0.5 frame/sec.

When using network communication as in this experiment, the data transfer delay has to be considered. As this system doesn't manage event time, the delay causes a different result between two sites. The delay between Japan and Germany was measured by using UNIX command ping. Ping command result was 300ms (round- 
trip time). At this speed, it is no necessary for the user to wait for the processing completion. However, if both users in each site interact with a 3D object simultaneously, each site's simulation result will be different. Therefore, each user conducts their procedures in turns.

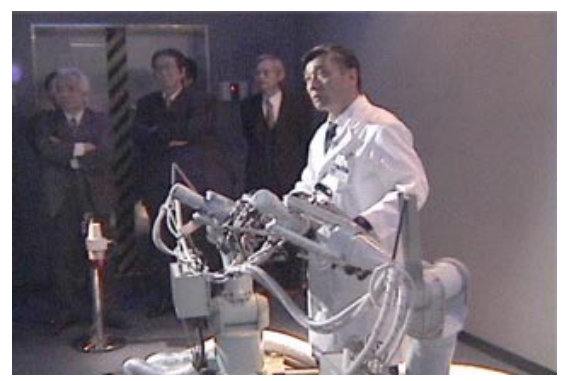

a. Japanese site

\section{Results}

For the experiment, a simulated hepatectomy was chosen. Fig. 6 shows a scene of the experiment at the both sites. Surgeons in each location palpated the patient's abdominal skin (Fig.7a) and discussed an incision position while observing location of the tumor and vascularity of the liver by changing skin and liver surface transparency. While a Japanese surgeon widened the incision line using a surgical tool, surgeons in Germany

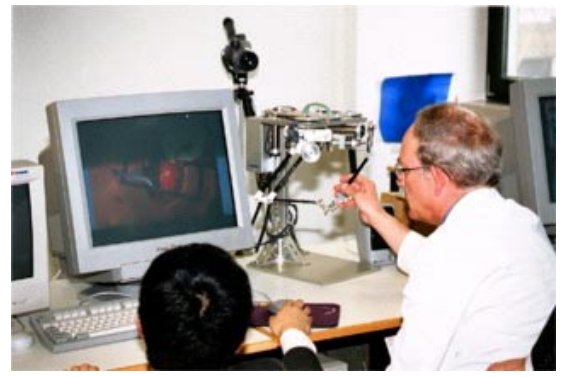

b. German site

Fig. 7. Scene of the experiment at both sites made an incision on the skin surface (Fig.7b). After widening, they palpated the exposed liver and deliberated upon an incision to the liver (Fig.7c). Finally, a German surgeon made an incision to the liver to complete the hepatectomy (Fig.7d).

In this system, the display's frame rate was 6-7 frame/sec. However, considering the surgeons action in surgical simulation, the frame rate was acceptable for the simulation. The two surgeons in Japan and Germany also had no comment on the frame rate.

Both surgeons evaluated this system and the experiment. The Japanese surgeon commented that he felt in close proximity the German surgeon and didn't sense any delay in the operation. On the other hand, the German surgeon observed that he could discuss surgery procedures in detail with the Japanese surgeon in order to find a solution to the surgical problem.

\section{Discussion}

We have demonstrated the virtual surgery with two surgeons while sharing identical tactile sensations over a long distance. It was possible to obtain real-time tele-virtual surgery without a large capacity communication infrastructure.

However, this system has a limitation. Both sites operated on a $3 \mathrm{D}$ object in turn, due to time delays when communicating event signals. Different results were caused by the delay between two sites. Therefore, evaluating the effects of time delay on the 

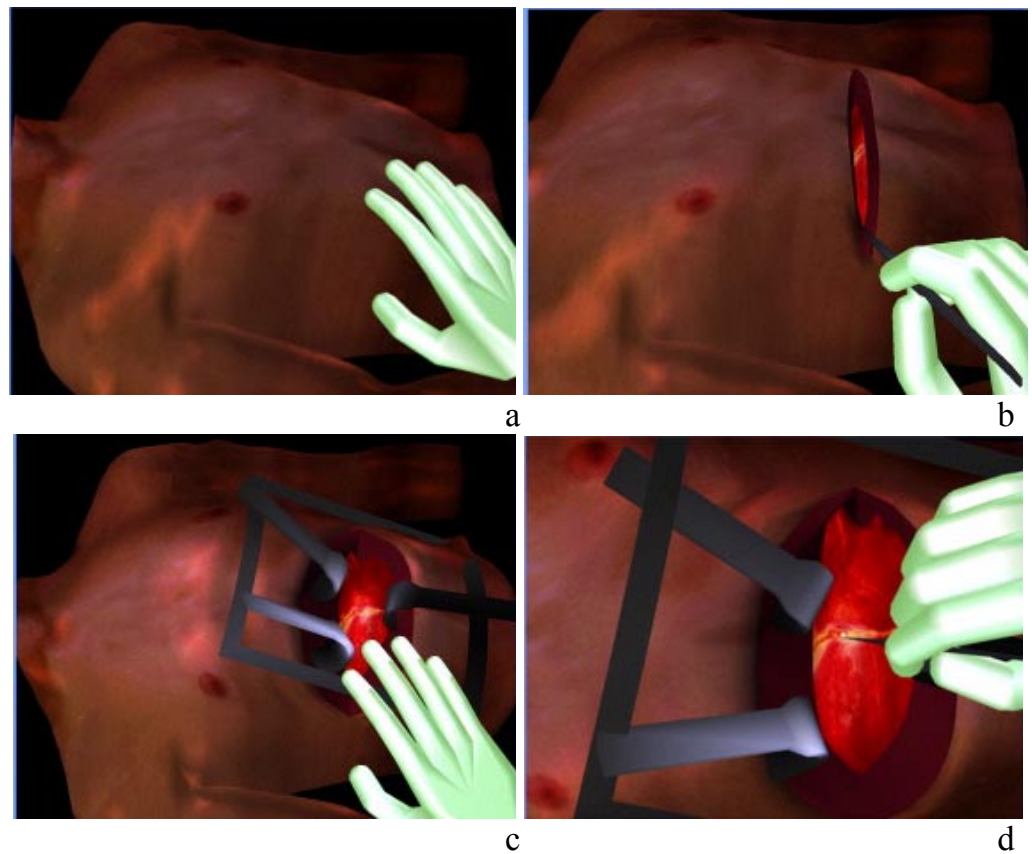

Fig. 8. Images of hepatectomy simulation. a) palpating the abdominal skin, b) making an incision to the abdominal skin, c) palpating the exposed liver, d) making an incision to the liver

surgical simulation and develop a system which enable users to manipulate a 3D object simultaneously was needed. The revised system will be the basis of the telesurgery system.

A 3D model structure caused the drawbacks of the force feedback function. Elasticity of this model's is configured only on the surface, and it doesn't depend on an organ's internal structure. Now, we are developing another 3D model "Sphere filled model". This model is reconstructed as a surface model filled with small element spheres with which a force acting on the internal structure can be calculated. We can improve tactile sensations by applying this model.

\section{References}

1. Suzuki. N, Takatsu. A, Kita. K, Tanaka. T, Inaba. R, Fukui. K: Development of a 3D image simulation system for organ and soft tissue operations.: Abstract of the World Congress on Medical Physics and Biomedical Engineering 1994; 39a: 609.

2. Robb RA, Hanson DP: The ANALYZE software system for visualization and analysis in surgery simulation. In: Computer Integrated Surgery, Eds. Steve Lavalle, Russ Taylor, Greg Burdea and Ralph Mosges, MIT Press, 1995, pp.175-190.

3. Robb RA, Cameron B: Virtual Reality Assisted Surgery Program. In: Interactive Technology and the New Paradigm for Healthcare, Eds., R. Satava, et al., Vol. 18, 1995, pp.309-321 
4. Kikinis R, Langham Gleason P, Jolesz FA: Surgical planning using computer-assisted threedimensional reconstructions. In: Computer Integrated Surgery, Eds. Russel Taylor, Stephane Lavallee, Grigore Burdea, and Ralph Mosges. MIT Press, 1995, pp.147-154.

5. N. Suzuki, A. Hattori, A. Takatsu: "Medical virtual reality system for surgical planning and surgical support", J. comput. Aided Surg., 54-59, 1(2), 1995.

6. N. Suzuki, A. Hattori, S. Kai, T. Ezumi, A. Takatsu: "Surgical planning system for soft tissues using virtual reality", MMVR5, Eds: K.S. Morgan et al., pp.159-163, IOS Press, 1997.

7. D. Terzopoulos and K. Fleischer: "Modeling inelastic deformation: Viscoelasticity, plasticity, fracture", Computer Graphics, vol.22, NO.4, pp.269-278, 1988.

8. A. Norton, G. Turk, B. Bacon, J. Gerth, and P. Sweeney: "Animation of fracture by physical modeling", The Visual Computer, vol.7, pp.210-219, 1991.

9. H. Delingette: "Simplex Meshes: a General Representation for 3D Shape Reconstruction", Technical Report 2214, INRIA, Sophia-Antipolis, France, 1994. 